\title{
Global Asymptotical Stability of Neutral-Type Neural Networks with $D$-Operator and Mixed Delays
}

\author{
Qing Yang, Bo Du $(\mathbb{D}$, and Xiwang Cheng \\ Department of Mathematics, Huaiyin Normal University, Huaian, Jiangsu 223300, China \\ Correspondence should be addressed to Bo Du; dubo7307@163.com
}

Received 23 October 2019; Revised 2 December 2019; Accepted 14 December 2019; Published 22 January 2020

Academic Editor: Quanxin Zhu

Copyright (c) 2020 Qing Yang et al. This is an open access article distributed under the Creative Commons Attribution License, which permits unrestricted use, distribution, and reproduction in any medium, provided the original work is properly cited.

In this manuscript, we investigate the stability problems of neutral-type neural networks with $D$-operator and mixed delays. Some sufficient conditions are obtained for guaranteeing the existence, uniqueness, and global asymptotical stability of periodic solutions to the considered neural networks. Finally, a numerical example is performed to illustrate the theoretical results.

\section{Introduction}

In this article, we consider the following neutral neural networks with $D$-operator and mixed delays:

$$
\begin{cases}(A x)^{\prime}(t)=-B \alpha(x(t))+K f(x(t))+U g(x(t-\tau(t)))+W \int_{t-\mu(t)}^{t} h(s) \mathrm{d} s+I(t), & t>0, \\ x(s)=\phi(s), & s \in[-\rho, 0]\end{cases}
$$

where $x(t)=\left(x_{1}(t), \ldots, x_{n}(t)\right)^{\top}$ corresponds to the state of the ith unit at time $t$ and $(A x)(t)=\left(\left(A_{1} x_{1}\right)\right.$ $\left.(t), \ldots,\left(A_{n} x_{n}\right)(t)\right)^{\top}$, which is defined by

$$
(A x)(t)=x(t)-C x(t-\sigma), \quad t \in \mathbb{R}
$$

where $\sigma>0$ is a constant delay, $C=\operatorname{diag}\left\{c_{1}, \ldots, c_{n}\right\}$ with $\left|c_{i}\right|<1 ; \alpha(x(t))=(A x)(t)$ denotes the behaved function; the functions $f(x(\cdot))=\left(f_{1}\left(x_{1}(\cdot)\right), \ldots, f_{n}\left(x_{n}(\cdot)\right)\right)^{\top}, g(x(\cdot))=$ $\left(g_{1}\left(x_{1}(\cdot)\right), \ldots, g_{n}\left(x_{n}(\cdot)\right)\right)^{\top}, \quad$ and $h(x(\cdot))=\left(h_{1}\left(x_{1}(\cdot)\right)\right.$ $\left., \ldots, h_{n}\left(x_{n}(\cdot)\right)\right)^{\top}$ denote the neuron activations; $\tau(t)$ and $\mu(t)$ are the discrete time-varying delay and the distributed time-varying delay, respectively, which satisfy $(0 \leq \tau(t) \leq \tau, 0 \leq \mu(t) \leq \mu)$, where $\tau$ and $\mu$ are positive constants; and $\rho=\max \{\sigma, \tau, \mu\}$, for $\phi \in C\left([-\rho, 0], \mathbb{R}^{n}\right)$, and the norm is defined by $\|\phi\|_{\rho}=\sup _{-\rho \leq s \leq 0}|\phi(s)|$. The matrix $B=$ $\left(b_{i j}\right)_{n \times n}>0$ is a constant matrix, and $K=\left(k_{i j}\right)_{n \times n}>0, U=$ $\left(u_{i j}\right)_{n \times n}$, and $W=\left(w_{i j}\right)_{n \times n}$ are some unknown constant matrices. $I(t)$ denotes the exogenous inputs at time $t$.

Remark 1. The neutral-type operator A in (2) represents the $D$-operator form which was put forward by Hale [1]. Then, we give the following properties for the difference operator $\mathscr{A}$. Define the operator $\mathscr{A}$ on $C_{T}$ :

$$
\begin{aligned}
& \mathscr{A}: C_{T} \longrightarrow C_{T}, \\
& {[\mathscr{A} x](t)=x(t)-c x(t-\tau), \quad \forall t \in \mathbb{R},}
\end{aligned}
$$

where $C_{T}=\{x: x \in C(\mathbb{R}, \mathbb{R}), x(t+T) \equiv x(t)\}$ and $c$ is a constant. 
Lemma 1 (see $[2,3]$ ). When $|c| \neq 1$, then $\mathscr{A}$ has a unique continuous bounded inverse $\mathscr{A}^{-1}$ satisfying

$$
\begin{aligned}
& {\left[\mathscr{A}^{-1} f\right](t)= \begin{cases}\sum_{j \geq 0} c^{j} f(t-j \tau), & \text { if }|c|<1, \forall f \in C_{T}, \\
-\sum_{j \geq 1} c^{-j} f(t+j \tau), & \text { if }|c|>1, \forall f \in C_{T},\end{cases} } \\
& \left|\mathscr{A}^{-1}\right| \leq \frac{1}{|1-| c||} .
\end{aligned}
$$

When the neutral-type operator $\mathscr{A}$ is defined on continuous function space $C(\mathbb{R}, \mathbb{R})$, we have the following lemma:

Lemma 2 (see [3]). If $|c|<1$, then the inverse of difference operator $\mathscr{A}$ denoted by $\mathscr{A}^{-1}$ exists, and

$$
\left|\mathscr{A}^{-1}\right| \leq \frac{1}{1-c} \text {. }
$$

Proof. The proof of Lemma 2 is similar to the proof in [3]. For the convenience of the reader, we provide a detailed proof. Let $B x(t)=c x(t-\tau)$, then $|B|=|c|<1$. Thus, $\mathscr{A}^{-1}=$ $(I-B)^{-1}$ exists, and

$$
\left|\mathscr{A}^{-1}\right|=\left|(I-B)^{-1}\right| \leq \frac{1}{1-|c|} .
$$

Remark 2. Since the matrices $K, U$, and $W$ in (1) are uncertain matrices, system (1) is interval neural networks. In the real world, the dynamic systems are often destroyed by various inevitable factors, such as parameter fluctuation, external disturbance, and random disturbance. Hence, the research for interval neural networks has important practical application value. There are lots of results for interval neural networks, e.g., $[4,5]$.

Let us recall the research for the stability problems of neural networks. Zhang [6] studied the stability problem of periodic solutions for a time-varying recurrent cellular neural network with time delays and impulses. Then, Zhang and Wang [7] further investigated dynamic properties of periodic solutions for high-order Hopfield neural networks with time delays and impulses. The models in $[6,7]$ are nonneutral-type models which are different from the model in the present paper. In [8], periodic oscillation problems of discrete-time bidirectional associative memory neural networks have been studied by employing the theory of coincidence degree and Halanay-type inequality technique. In the present paper, we obtain the existence, uniqueness, and global asymptotical stability of periodic solutions by using LMI approach, Lyapunov function, and a blend of matrix theory which are different from the methods in [8]. Zhu and Cao [9, 10] studied two different types of stochastic neural networks which are not neutral-type neural networks and are different from the model in the present paper. In $[11,12]$, the authors considered the following two kinds of neutral-type neural system:

$$
\begin{aligned}
\left\{\begin{array}{l}
\left(x_{i}\right)^{\prime}(t)=-a_{i}(t) x_{i}(t)+\sum_{j=1}^{n}\left[b_{i j}(t) f_{j}\left(t, x_{j}(t)\right)+d_{i j}(t) g_{j}\left(t, x_{j}^{\prime}\left(t-\tau_{i j}(t)\right)\right)\right]+I_{i}(t), \\
x_{i}(t)=\phi_{i}(t), t \in[-\tau, 0], \quad i=1,2, \ldots, n,
\end{array}\right. \\
y^{\prime}(t)=-A y(t)+B g(y(t))+C g(y(t-\tau(t)))+D y^{\prime}(t-h(t)), \quad t \neq t_{k}, \\
\Delta y(t)=I_{k}(y(t)), \quad t=t_{k}, \\
y\left(t_{0}^{+}+s\right)=\phi(s), \quad s \in\left[t_{0}-\rho, t_{0}\right], \quad k \in \mathbb{N} .
\end{aligned}
$$

Liu et al. [13] studied a class of Markovian jumping neutral-type neural networks with mode-dependent mixed time delays:

$$
\begin{aligned}
\dot{x}(t)= & E(r(t)) \dot{x}\left(t-\tau_{1, r(t)}\right)-A(r(t)) x(t)-B(r(t)) f(x(t)) \\
& +c(r(t)) g\left(x\left(t-\tau_{2, r(t)}\right)\right)+D(r(t)) \int_{t-\tau_{3, r(t)}}^{t-\tau_{4, r(t)}} h(x(s)) \mathrm{d} s .
\end{aligned}
$$

By the above work, we note that the neutral character in neural networks shows by the nonlinear term $g\left(t, x^{\prime}(t-\right.$ $\tau(t)))$ or differential operator $\left(x^{\prime}(t-\tau(t))\right)$. In this paper, we will study the neutral-type neural networks when the neutral term has the $D$-operator form which is shown by
$A x(t)$. For more references about neutral-type neural networks with mixed delays, see $[14,15]$.

To best knowledge of the authors, few authors have studied the global asymptotical stability problems of periodic solutions to neutral-type neural networks with mixed delays. The main areas of challenge are as follows: (1) since system (1) contains neutral-type operator $A$, constructing a viable Lyapunov-Krasovskii functional seems to be very difficult; (2) as the mixed delays exist in system (1), the corresponding LMI approach becomes more complicated since the LMI approach is required to reflect mixed delay's influence; and (3) it is extraordinary to establish a unified framework to dispose of the uncertain matrices, the neutral-type terms, and mixed delays' influence. Therefore, the main purpose of this article is to try for the first time to address the challenges listed. 
In this paper, we will study global asymptotical stability of interval neural networks for a neutral-type neural network with mixed delays. Note that system (1) contains uncertain matrices, the neutral-type terms, and mixed delays that are all dependent on the properties of the neutral operator.

Remark 3. The main purpose of this paper is to obtain some sufficient conditions for guaranteeing asymptotic stability of system (1). We develop LMI approach to answer the addressed challenges. A simulation example is given to show the usefulness of the main results in the present paper. There are three aspects to the contribution of this paper: (1) For using the LMI method, we have to take into account the properties of the neutral-type operator. (2) Unlike the most existing results, we develop a new unified framework to deal with global asymptotical stability of interval neural networks by LMI approach, Lyapunov function, and a blend of matrix theory which may be of independent interest. It is worth pointing out that our main methods are also important for the case of nonneutral system with constant delays. (3) This article uses some new inequality techniques. In particular, using the properties of the neutraltype operator, we construct an appropriate LyapunovKrasovskii functional to handle the considered system.

The following sections are organized as follows: Section 2 gives some preliminaries including some useful lemmas and definitions. In Section 3, we obtain some sufficient conditions for existence, uniqueness, and global asymptotic stability of periodic solution to system (1). In Section 4, a numerical example verifies the accuracy of the results in the present paper.

\section{Some Preliminaries}

Throughout the paper, $\Lambda=\{1,2, \ldots, n\}$, and $\mathbb{R}^{n}$ and $\mathbb{R}^{n \times m}$ denote the $n$-dimensional Euclidean space and the set of all $n \times m$ real matrices, respectively. The superscript " $T$ " represents the matrix transposition. $A>0$ (or $A<0$ ) denotes that $A$ is a symmetric and positive definite (or negative definite) matrix. $|z|$ denotes the Euclidean norm of a vector $z$ and $\|A\|$ denotes the induced norm of the matrix $A$, that is, $\|A\|=\sqrt{\lambda_{\max }\left(A^{\top} A\right)}$, where $\lambda_{\max }(\cdot)$ means the largest eigenvalue of $A$. If their dimensions are not explicitly stated, they are assumed to be compatible for algebraic operations.

Lemma 3 (see [16]). For any vectors with $x, y \in \mathbb{R}^{n}$, the inequality

$$
\pm 2 x^{\top} y \leq x^{\top} \mathscr{M} x+y^{\top} \mathscr{M} y
$$

holds, where $\mathscr{M}$ is any $n \times n$ matrix with $\mathscr{M}>0$.

Lemma 4 (see [17]). Let $p, q, \gamma, \tau$, and $\sigma$ be the positive constants, and the function $f \in P C\left(\mathbb{R}, \mathbb{R}_{+}\right)$satisfies the scalar impulsive differential inequality:

$$
\begin{cases}D^{+} f(t) \leq-p f(t)+q \sup _{t-\tau \leq s \leq t} f(s)+\int_{0}^{\sigma} r(s) f(t-s) \mathrm{d} s, & t \neq t_{k}, t \geq 0 \\ f\left(t_{k}\right) \leq f\left(t_{k}^{-}\right), & k \in \mathbb{Z}_{+},\end{cases}
$$

where $r(\cdot) \in P C\left([0, \sigma], \mathbb{R}^{+}\right) . \quad$ Assume that $p>q+\gamma \int_{0}^{\sigma} r(s) d s$, then

$$
f(t) \leq \bar{f}(0) e^{-\lambda t}, \quad t \geq 0,
$$

where $\bar{f}(0)=\sup _{-\rho \leq s \leq 0} f(s), \rho=\max \{\sigma, \tau\}$, and $\lambda>0$ satisfies $\lambda<p-q e^{\lambda \tau}-\gamma \int_{0}^{\sigma} r(s) e^{\lambda s} d s$.

Remark 4. When $f\left(t_{k}\right)=f\left(t_{k}^{-}\right)$, then $f(t)$ is a continuous function on $[0, \infty)$, and the results of Lemma 4 also hold.

Throughout this paper, the following assumptions are needed:

$\left(H_{1}\right)$ For $i \in \Lambda$, the neuron activation functions in (1) satisfy

$$
\begin{gathered}
l_{i}^{-} \leq \frac{f_{i}\left(s_{1}\right)-f_{i}\left(s_{2}\right)}{s_{1}-s_{2}} \leq l_{i}^{+}, \\
\sigma_{i}^{-} \leq \frac{g_{i}\left(s_{1}\right)-g_{i}\left(s_{2}\right)}{s_{1}-s_{2}} \leq \sigma_{i}^{+}, \\
v_{i}^{-} \leq \frac{h_{i}\left(s_{1}\right)-h_{i}\left(s_{2}\right)}{s_{1}-s_{2}} \leq v_{i}^{+},
\end{gathered}
$$

\begin{abstract}
where $l_{i}^{-}, l_{i}^{+}, \sigma_{i}^{-}, \sigma_{i}^{+}, v_{i}^{-}$, and $v_{i}^{+}$are the real constants and they may be positive, zero, or negative.
\end{abstract}

$\left(H_{2}\right) \quad \tau(t), \mu(t)$, and $I(t)$ are all continuously $T$-periodic functions on $[0, \infty)$.

\section{Main Results}

Assume that the unknown constant matrices $K, U$, and $W$ satisfy

$$
\begin{gathered}
K \in \mathbb{K}, \\
U \in \mathbb{U}, \\
W \in \mathbb{W},
\end{gathered}
$$

where

$$
\begin{aligned}
& \mathbb{K}=[\underline{K}, \bar{K}]=:\left\{\left(k_{i j}\right)_{n \times n} \mid \underline{k}_{i j} \leq k_{i j} \leq \bar{k}_{i j}, i, j \in \Lambda\right\}, \\
& \mathbb{U}=[\underline{U}, \bar{U}]=:\left\{\left(u_{i j}\right)_{n \times n} \mid \underline{u}_{i j} \leq u_{i j} \leq \bar{u}_{i j}, i, j \in \Lambda\right\}, \\
& \mathbb{W}=[\underline{W}, \bar{B}]=:\left\{\left(w_{i j}\right)_{n \times n} \mid \underline{w}_{i j} \leq w_{i j} \leq \bar{w}_{i j}, i, j \in \Lambda\right\},
\end{aligned}
$$


with

$$
\begin{aligned}
& \underline{K}=\left(\underline{k}_{i j}\right)_{n \times n^{\prime}} \\
& \bar{K}=\left(\bar{k}_{i j}\right)_{n \times n^{\prime}} \\
& \underline{U}=\left(\underline{u}_{i j}\right)_{n \times n^{\prime}} \\
& \bar{U}=\left(\bar{u}_{i j}\right)_{n \times n^{\prime}} \\
& \underline{W}=\left(\underline{w}_{i j}\right)_{n \times n^{\prime}} \\
& \bar{W}=\left(\bar{w}_{i j}\right)_{n \times n} .
\end{aligned}
$$

$$
\begin{aligned}
& \Delta=:\left\{\operatorname{diag}\left(\delta_{11}, \ldots, \delta_{1 n}, \ldots, \delta_{n 1}, \ldots, \delta_{n n}\right) \in \mathbb{R}^{n^{2} \times n^{2}}, \quad\left|\delta_{i j}\right| \leq 1, i, j \in \Lambda\right\} \\
& K_{0}=\frac{K+\bar{K}}{2} \\
& U_{0}=\frac{U}{2}, \\
& W_{0}=\frac{W+\bar{W}}{2} \\
& \left(\eta_{i j}\right)_{n \times n}=\frac{\bar{K}-\underline{K}}{2} \\
& \left(\iota_{i j}\right)_{n \times n}=\frac{\bar{U}-\underline{U}}{2}, \\
& \left(\kappa_{i j}\right)_{n \times n}=\frac{\bar{W}-\underline{W}}{2} \\
& \Gamma_{1}=\left\{\sqrt{\eta_{11}} e_{1}, \ldots, \sqrt{\eta_{1 n}} e_{1}, \ldots, \sqrt{\eta_{n 1}} e_{n}, \ldots, \sqrt{\eta_{n n}} e_{n}\right\}_{n \times n^{2}}, \\
& \Gamma_{2}=\left\{\sqrt{l_{11}} e_{1}, \ldots, \sqrt{l_{1 n}} e_{1}, \ldots, \sqrt{l_{n 1}} e_{n}, \ldots, \sqrt{l_{n n}} e_{n}\right\}_{n \times n^{2}} \\
& \Gamma_{3}=\left\{\sqrt{\kappa_{11}} e_{1}, \ldots, \sqrt{\kappa_{1 n}} e_{1}, \ldots, \sqrt{\kappa_{n 1}} e_{n}, \ldots, \sqrt{\kappa_{n n}} e_{n}\right\}_{n \times n^{2}} \text {, } \\
& \Upsilon_{1}=\left\{\sqrt{\eta_{11}} e_{1}, \ldots, \sqrt{\eta_{1 n}} e_{1}, \ldots, \sqrt{\eta_{n 1}} e_{n}, \ldots, \sqrt{\eta_{n n}} e_{n}\right\}_{n^{2} \times n}^{\top}, \\
& \Upsilon_{2}=\left\{\sqrt{l_{11}} e_{1}, \ldots, \sqrt{l_{1 n}} e_{1}, \ldots, \sqrt{l_{n 1}} e_{n}, \ldots, \sqrt{l_{n n}} e_{n}\right\}_{n^{2} \times n}^{\top}, \\
& \Upsilon_{3}=\left\{\sqrt{\kappa_{11}} e_{1}, \ldots, \sqrt{\kappa_{1 n}} e_{1}, \ldots, \sqrt{\kappa_{n 1}} e_{n}, \ldots, \sqrt{\kappa_{n n}} e_{n}\right\}_{n^{2} \times n}^{\top},
\end{aligned}
$$

where $e_{i} \in \mathbb{R}^{n}$ and $i \in \Lambda$ denotes the column vector with $i$ th element to be 1 and others to be 0 . Then, system (1) can be rewritten as

$$
\mid \begin{array}{ll}
(A x)^{\prime}(t)=-B \alpha(x(t))+\left(K_{0}+\Gamma_{1} \Delta_{1} \Upsilon_{1}\right) f(x(t))+\left(U_{0}+\Gamma_{2} \Delta_{2} \Upsilon_{2}\right) g(x(t-\tau(t)))+\left(W_{0}+\Gamma_{3} \Delta_{3} \Upsilon_{3}\right) \int_{t-\mu(t)}^{t} h(s) \mathrm{d} s+I(t), & t>0, \\
x(s)=\phi(s), & s \in[-\rho, 0],
\end{array}
$$


i.e.,

$$
\begin{cases}(A x)^{\prime}(t)=-B \alpha(x(t))+K_{0} f(x(t))+U_{0} g(x(t-\tau(t)))+W_{0} \int_{t-\mu(t)}^{t} h(s) \mathrm{d} s+\Theta \Psi(t)+I(t), & t>0, \\ x(s)=\phi(s), & s \in[-\rho, 0],\end{cases}
$$

where $\Theta=\left\{\Gamma_{1}, \Gamma_{2}, \Gamma_{3}\right\}_{n \times 3 n^{2}}$,

$$
\Psi(t)=\left(\begin{array}{ccc}
\Delta_{1} \Upsilon_{1} & 0 & 0 \\
0 & \Delta_{2} \Upsilon_{2} & 0 \\
0 & 0 & \Delta_{3} \Upsilon_{3}
\end{array}\right)_{3 n^{2} \times n} \times\left(\begin{array}{c}
f(x(t)) \\
g(x(t-\tau(t))) \\
\int_{t-\mu(t)}^{t} h(s) \mathrm{d} s
\end{array}\right)
$$

$\Delta_{i} \in \Delta, \quad i=1,2,3$.

Now, we present the main results of the present paper.

Theorem 1. Assume that the assumptions $\left(H_{1}\right)$ and $\left(H_{2}\right)$ hold. Then, system (1) has a global asymptotically stable periodic solution if there exist three constants $\varepsilon_{i}>0, i=1,2,3$, $n \times n$ diagonal matrices $P>0$, and $Q_{i}>0, i=1,2,3$, and three $n^{2} \times n^{2}$ diagonal matrices $Q_{i}>0, i=4,5,6$, such that the following inequalities hold:

$$
\left\{\begin{array}{l}
\Xi \geq 0, \\
\varepsilon_{2} P-\sum_{i=1}^{n} \frac{1}{\left.|1-| c_{i}\right|^{2}} L^{g} Q_{2} L^{g}-\sum_{i=1}^{n} \frac{1}{|1-| c_{i}||^{2}} L^{g} \Upsilon_{1}^{\top} Q_{5} \Upsilon_{1} L^{g} \geq 0, \\
\varepsilon_{3} P-\sum_{i=1}^{n} \frac{1}{|1-| c_{i}||^{2}} L^{h} Q_{3} L^{h}-\sum_{i=1}^{n} \frac{1}{|1-| c_{i}||^{2}} L^{h} \Upsilon_{3}^{\top} Q_{6} \Upsilon_{3} L^{h} \geq 0, \\
\varepsilon_{1}-\varepsilon_{2}-\varepsilon_{3} \mu>0,
\end{array}\right.
$$

where

$$
\Xi=\left(\begin{array}{ccccc}
\Xi_{1} & P K_{0} & P U_{0} & P W_{0} & P \Theta \\
* & Q_{1} & 0 & 0 & 0 \\
* & * & Q_{2} & 0 & 0 \\
* & * & * & Q_{3} & 0 \\
* & * & * & * & \Xi_{2}
\end{array}\right),
$$

$$
\Xi_{1}=2 P B-\varepsilon_{1} P-\sum_{i=1}^{n} \frac{1}{|1-| c_{i}||^{2}} L^{f} Q_{1} L^{f}-\sum_{i=1}^{n} \frac{1}{|1-| c_{i}||^{2}} L^{f} \Upsilon_{1}^{\top} Q_{4} \Upsilon_{1} L^{f} \text {, }
$$

$$
\Xi_{2}=\left(\begin{array}{ccc}
Q_{4} & 0 & 0 \\
* & Q_{5} & 0 \\
* & * & Q_{6}
\end{array}\right)
$$$$
L^{f}=\operatorname{diag}\left(l_{1}, \ldots, l_{n}\right), l_{j}=\max \left\{\left|l_{j}^{-}\right|,\left|l_{j}^{+}\right|, j \in \Lambda\right\},
$$$$
L^{g}=\operatorname{diag}\left(\sigma_{1}, \ldots, \sigma_{n}\right), \sigma_{j}=\max \left\{\left|\sigma_{j}^{-}\right|,\left|\sigma_{j}^{+}\right|, j \in \Lambda\right\},
$$$$
L^{h}=\operatorname{diag}\left(v_{1}, \ldots, v_{n}\right), v_{j}=\max \left\{\left|v_{j}^{-}\right|,\left|v_{j}^{+}\right|, j \in \Lambda\right\} .
$$

Proof. Let $x(t, \phi)$ be an arbitrary solution of system (1) with initial value $(0, \phi)$. Define $y(t, \psi)=x(t+T, \phi)$, where $T>0$ is a constant and $\psi=x(s+T, \phi),-\rho \leq s \leq 0$. Then $y(t, \psi)$ is a solution of system (1) with initial value $(0, \psi)$. Let $z(t)=y(t, \psi)-x(t, \phi)$. In view of system (18), we have

$$
\left\{\begin{array}{l}
(A x)^{\prime}(t)=-B \alpha[y(t)-x(t)]+K_{0}[f(y(t))-f(x(t))]+U_{0}[g(y(t-\tau(t)))-g(x(t-\tau(t)))] \\
\quad+W_{0} \int_{0}^{\mu(t)}[h(y(t-s))-h(x(t-s))] \mathrm{d} s+\Theta \Psi_{z}(t), \quad t>0, \\
x(s)=\psi(s)-\phi(s), s \in[-\rho, 0],
\end{array}\right.
$$

where

$$
\begin{aligned}
\Psi_{z}(t)= & \left(\begin{array}{ccc}
\Delta_{1} \Upsilon_{1} & 0 & 0 \\
0 & \Delta_{2} \Upsilon_{2} & 0 \\
0 & 0 & \Delta_{3} \Upsilon_{3}
\end{array}\right)_{3 n^{2} \times n} \\
& \times\left(\begin{array}{c}
f(y(t))-f(x(t)) \\
g(y(t-\tau(t)))-g(x(t-\tau(t))) \\
\int_{0}^{\mu(t)}[h(y(t-s))-h(x(t-s))] \mathrm{d} s
\end{array}\right) .
\end{aligned}
$$

Construct the following Lyapunov function:

$$
V(t)=[(A z)(t)]^{\top} P[(A z)(t)], \quad t \geq 0 .
$$

Derivation of the above Lyapunov function along the solution of (22) gives 


$$
\begin{aligned}
D^{+} V(t)= & 2[(A z)(t)]^{\top} P\left\{-B \alpha[y(t)-x(t)]+K_{0}[f(y(t))\right. \\
& -f(x(t))]+U_{0}[g(y(t-\tau(t)))-g(x(t-\tau(t)))] \\
& \left.+W_{0} \int_{0}^{\mu(t)}[h(y((t-s)))-h(x(t-s))] \mathrm{d} s+\Theta \Psi_{z}(t)\right\} \\
= & -2[(A z)(t)]^{\top} P B[(A z)(t)]+2[(A z)(t)]^{\top} P K_{0}[f(y(t)) \\
& -f(x(t))]+2[(A z)(t)]^{\top} P U_{0}[g(y(t-\tau(t))) \\
& -g(x(t-\tau(t)))]+2[(A z)(t)]^{\top} W_{0} \\
& \cdot \int_{0}^{\mu(t)}[h(y(t-s))-h(x(t-s))] \mathrm{d} s+2[(A z)(t)]^{\top} \Theta \Psi_{z}(t) .
\end{aligned}
$$

We calculate every term of $D^{+} V(t)$. It follows from the assumption $\left(H_{1}\right)$, Lemma 2 , and Lemma 3 that

$$
\begin{aligned}
& 2[(A z)(t)]^{\top} P K_{0}[f(y(t))-f(x(t))] \\
& \leq[(A z)(t)]^{\top} P K_{0} Q_{1}^{-1} K_{0}^{\top} P[(A z)(t)] \\
& \quad+[g(y(t-\tau(t)))-g(x(t-\tau(t)))]^{\top} Q_{2} \\
& \quad \cdot[g(y(t-\tau(t)))-g(x(t-\tau(t)))] \\
& \leq[(A z)(t)]^{\top} P K_{0} Q_{1}^{-1} K_{0}^{\top} P[(A z)(t)] \\
& \quad+\sum_{i=1}^{n} \frac{1}{\left.|1-| c_{i}\right|^{2}}[(A z)(t)]^{\top} L^{f} Q_{1} L^{f}[(A z)(t)],
\end{aligned}
$$

$$
\begin{aligned}
& 2 {[(A z)(t)]^{\top} P U_{0}[g(y(t-\tau(t)))-g(x(t-\tau(t)))] } \\
& \leq {[(A z)(t)]^{\top} P U_{0} Q_{2}^{-1} U_{0}^{\top} P[(A z)(t)] } \\
&+[g(y(t-\tau(t)))-g(x(t-\tau(t)))]^{\top} Q_{2} \\
& \cdot[g(y(t-\tau(t)))-g(x(t-\tau(t)))] \\
& \leq {[(A z)(t)]^{\top} P U_{0} Q_{2}^{-1} U_{0}^{\top} P[(A z)(t)] } \\
&+\sum_{i=1}^{n} \frac{1}{\left.|1-| c_{i}\right|^{2}}[(A z)(t-\tau(t))]^{\top} L^{g} Q_{2} L^{g} \\
& {[(A z)(t-\tau(t))], } \\
& 2[(A z)(t)]^{\top} W_{0} \int_{0}^{\mu(t)}[h(y(t-s))-h(x(t-s))] \mathrm{d} s \\
& \leq {[(A z)(t)]^{\top} P W_{0} Q_{3}^{-1} W_{0}^{\top} P[(A z)(t)] } \\
&+\left[\int_{0}^{\mu(t)}[h(y(t-s))-h(x(t-s))] \mathrm{d} s\right]^{\top} Q_{3} \\
& \cdot\left[\int_{0}^{\mu(t)}[h(y(t-s))-h(x(t-s))] \mathrm{d} s\right] \\
& \leq {[(A z)(t)]^{\top} P W_{0} Q_{3}^{-1} W_{0}^{\top} P[(A z)(t)] } \\
&+\int_{0}^{\mu(t)} \sum_{i=1}^{n} \frac{1}{11-\left.\left|c_{i}\right|\right|^{2}}[(A z)(t-v)]^{\top} L^{h} Q_{3} L^{h} \\
& \quad[(A z)(t-v)] \mathrm{d} t .
\end{aligned}
$$

Using the definition of $\Psi_{z}(t)$, assumption $\left(H_{1}\right)$, and Lemma 2, we have

$$
\begin{aligned}
& \Psi_{z}(t)^{\top}\left(\begin{array}{ccc}
Q_{4} & 0 & 0 \\
0 & Q_{5} & 0 \\
0 & 0 & Q_{6}
\end{array}\right) \Psi_{z}(t) \\
& =\left(\begin{array}{c}
f(x(t))-f(y(t)) \\
g(x(t-\tau(t)))-g(y(t-\tau(t))) \\
\int_{0}^{\mu(t)}[h(x(t-s))-h(y(t-s))] \mathrm{d} s
\end{array}\right)^{\top} \\
& \times\left(\begin{array}{ccc}
\Upsilon_{1}^{\top} \Delta_{1} Q_{4} \Delta_{1} \Upsilon_{1} & 0 & 0 \\
0 & \Upsilon_{2}^{\top} \Delta_{2} Q_{5} \Delta_{2} \Upsilon_{2} & 0 \\
0 & 0 & \Upsilon_{3}^{\top} \Delta_{3} Q_{6} \Delta_{3} \Upsilon_{3}
\end{array}\right) \\
& \times\left(\begin{array}{c}
f(x(t))-f(y(t)) \\
g(x(t-\tau(t)))-g(y(t-\tau(t))) \\
\int_{0}^{\mu(t)}[h(x(t-s))-h(y(t-s))] \mathrm{d} s
\end{array}\right) \\
& \leq \sum_{i=1}^{n} \frac{1}{|1-| c_{i}||^{2}}[(A z)(t)]^{\top} L^{f} \Upsilon_{1}^{\top} Q_{4} \Upsilon_{1} L^{f}[(A z)(t)] \\
& +\sum_{i=1}^{n} \frac{1}{\left.|1-| c_{i}\right|^{2}}[(A z)(t-\tau(t))]^{\top} L^{g} \Upsilon_{1}^{\top} Q_{5} \Upsilon_{1} L^{g}[(A z)(t-\tau(t))] \\
& +\int_{0}^{\mu(t)} \sum_{i=1}^{n} \frac{1}{|1-| c_{i}||^{2}}[(A z)(t-v)]^{\top} L^{h} \Upsilon_{3}^{\top} Q_{6} \Upsilon_{3} L^{h}[(A z)(t-v)] \mathrm{d} t .
\end{aligned}
$$

By (29), we have

$$
\begin{aligned}
& 2[(A z)(t)]^{\top} \Theta \Psi_{z}(t) \leq[(A z)(t)]^{\top} P \Theta \\
& \left(\begin{array}{ccc}
Q_{4}^{-1} & 0 & 0 \\
0 & Q_{5}^{-1} & 0 \\
0 & 0 & Q_{6}^{-1}
\end{array}\right) \Theta^{\top} P[(A z)(t)] \\
& +\Psi_{z}(t)^{\top}\left(\begin{array}{ccc}
Q_{4} & 0 & 0 \\
0 & Q_{5} & 0 \\
0 & 0 & Q_{6}
\end{array}\right) \Psi_{z}(t) \\
& \leq[(A z)(t)]^{\top}\left\{P \Theta\left(\begin{array}{ccc}
Q_{4}^{-1} & 0 & 0 \\
0 & Q_{5}^{-1} & 0 \\
0 & 0 & Q_{6}^{-1}
\end{array}\right) \Theta^{\top} P\right. \\
& \left.+\sum_{i=1}^{n} \frac{1}{|1-| c_{i}||^{2}} L^{f} \Upsilon_{1}^{\top} Q_{4} \Upsilon_{1} L^{f}\right\}[(A z)(t)] \\
& +\sum_{i=1}^{n} \frac{1}{|1-| c_{i}||^{2}}[(A z)(t-\tau(t))]^{\top} L^{g} \Upsilon_{1}^{\top} Q_{5} \Upsilon_{1} L^{g} \\
& {[(A z)(t-\tau(t))]} \\
& +\int_{0}^{\mu(t)} \sum_{i=1}^{n} \frac{1}{|1-| c_{i}||^{2}}[(A z)(t-v)]^{\top} L^{h} \Upsilon_{3}^{\top} Q_{6} \Upsilon_{3} L^{h} \\
& {[(A z)(t-v)] \mathrm{d} t .}
\end{aligned}
$$


Adding the terms on the right-hand side of (26)-(28) and (30) to (25), and using condition (20), we get

$$
\begin{aligned}
D^{+} V(t) \leq & {[(A z)(t)]^{\top}\left\{-2 P B+P K_{0} Q_{1}^{-1} K_{0}^{\top} P+\sum_{i=1}^{n} \frac{1}{|1-| c_{i}||^{2}} L^{f} Q_{1} L^{f}+P U_{0} Q_{2}^{-1} U_{0}^{\top} P+P W_{0} Q_{3}^{-1} W_{0}^{\top} P+P \Theta\right.} \\
& \left.\cdot\left(\begin{array}{ccc}
Q_{4}^{-1} & 0 & 0 \\
0 & Q_{5}^{-1} & 0 \\
0 & 0 & Q_{6}^{-1}
\end{array}\right) \Theta^{\top} P+\sum_{i=1}^{n} \frac{1}{|1-| c_{i}||^{2}} L^{f} \Upsilon_{1}^{\top} Q_{4} \Upsilon_{1} L^{f}\right\}[(A z)(t)] \\
& +\sum_{i=1}^{n} \frac{1}{\left.|1-| c_{i}\right|^{2}}[(A z)(t-\tau(t))]^{\top}\left[L^{g} Q_{2} L^{g}+L^{g} \Upsilon_{1}^{\top} Q_{5} \Upsilon_{1} L^{g}\right][(A z)(t-\tau(t))] \\
& +\int_{0}^{\mu(t)} \sum_{i=1}^{n} \frac{1}{|1-| c_{i}||^{2}}[(A z)(t-v)]^{\top}\left[L^{h} Q_{3} L^{h}+L^{h} \Upsilon_{3}^{\top} Q_{6} \Upsilon_{3} L^{h}\right][(A z)(t-v)] \mathrm{d} t \\
\leq & -\varepsilon_{1}[(A z)(t)]^{\top} P[(A z)(t)]+\varepsilon_{2}[(A z)(t-\tau(t))]^{\top} P[(A z)(t-\tau(t))] \\
& +\varepsilon_{3} \int_{0}^{\mu}(t)[(A z)(t-v)]^{\top} P[(A z)(t-v)] \mathrm{d} v \\
\leq & -\varepsilon_{1} V(t)+\varepsilon_{2} V(t-\tau(t))+\varepsilon_{3} \int_{0}^{\mu}(t) V(t-v) \mathrm{d} v,
\end{aligned}
$$

which together with Remark 4 yields

$$
V(t) \leq \widehat{V}_{\rho} e^{-\lambda t}, \quad t \geq 0,
$$

where $\widehat{V}_{\rho}=\sup _{s \in[-\rho, 0]} V(s)$ and $\lambda>0$ satisfies $\lambda<\varepsilon_{1}-$ $\varepsilon_{2} e^{\lambda \tau}-\varepsilon_{3}\left(e^{\lambda \mu} / \lambda\right)$. Thus,

$$
\begin{gathered}
\lambda_{\min }(P)|A z(t)|^{2} \leq V(t) \leq \lambda_{\max }(P) \max _{-\rho \leq s \leq 0}\left\{|A(\phi(s)-\psi(s))|^{2}\right\} e^{-\lambda t}, \quad t \geq 0, \\
|A z(t)| \leq \sqrt{\frac{\lambda_{\max }(P)}{\lambda_{\min }(P)}} \max _{-\rho \leq s \leq 0}\{|A(\phi(s)-\psi(s))|\} e^{-0.5 \lambda t}, \quad t \geq 0 .
\end{gathered}
$$

By (33) and Lemma 2, we have

$$
\begin{aligned}
|z(t)| & \leq \sqrt{\sum_{i=1}^{n} \frac{1}{\left.|1-| c_{i}\right|^{2}}|A z(t)|} \\
& \leq \sqrt{\sum_{i=1}^{n} \frac{1}{\left.|1-| c_{i}\right|^{2}} \sqrt{\frac{\lambda_{\max }(P)}{\lambda_{\min }(P)}} \max _{-\rho \leq s \leq 0}\{|A(\phi(s)-\psi(s))|\} e^{-0.5 \lambda t}, \quad t \geq 0,}
\end{aligned}
$$


i.e.,

$$
|y(t, \psi)-x(t, \phi)| \leq \sqrt{\sum_{i=1}^{n} \frac{\lambda_{\max }(P)}{|1-| c_{i}||^{2} \lambda_{\min }(P)}} \max _{-\rho \leq s \leq 0}\{|A(\phi(s)-\psi(s))|\} e^{-0.5 \lambda t}, \quad t \geq 0 .
$$

Let

$$
\begin{aligned}
X_{m}(t)= & x(t, \phi)+\sum_{k=1}^{m}[x(t+k T, \phi)-x \\
& \cdot(t+(k-1) T, \phi)], \quad t \geq 0, m \in Z_{+} .
\end{aligned}
$$

We show that $X_{m}(t)$ is uniformly convergent. For $s \in Z_{+}$, by (35), we have

$$
\begin{aligned}
\left|X_{m+s}(t)-X_{m}(t)\right| & =\left|\sum_{k=m+1}^{m+s}[x(t+k T, \phi)-x(t+(k-1) T, \phi)]\right| \\
& \leq \sum_{k=m+1}^{m+s}|x(t+k T, \phi)-x(t+(k-1) T, \phi)| \\
& =\sum_{k=m+1}^{m+s}|y(t+(k-1) T, \psi)-x(t+(k-1) T, \phi)| \\
& \leq \sqrt{\sum_{i=1}^{n} \frac{\lambda_{\max }(P)}{\left.|1-| c_{i}\right|^{2} \lambda_{\min }(P)} \max _{-\rho \leq s \leq 0}\{|A(\phi(s)-\psi(s))|\} e^{-0.5 \lambda t} \sum_{k=m+1}^{m+s} e^{-0.5 \lambda(k-1) T}} \\
& \leq \sqrt{\sum_{i=1}^{n} \frac{\lambda_{\max }(P)}{\left.|1-| c_{i}\right|^{2} \lambda_{\min }(P)} \max _{-\rho \leq s \leq 0}\{|A(\phi(s)-\psi(s))|\} \frac{e^{-0.5 \lambda T m}}{1-e^{-0.5 \lambda T}}}
\end{aligned}
$$

It is obvious that the function sequence $\left\{X_{m}(t)\right\}_{m=1}^{\infty}$ is uniformly convergent by the Cauchy convergence criterion. In addition,

$$
\begin{aligned}
x(t+m T, \phi) & =x(t, \phi)+\sum_{k=1}^{m}[x(t+k T, \phi)-x(t+(k-1) T, \phi)] \\
& =X_{m}(t) .
\end{aligned}
$$

Hence, $x(t+m T, \phi)$ is also uniformly convergent, which implies that

$$
\begin{aligned}
\lim _{m \longrightarrow \infty} & x(t+m T, \phi)=x_{0}(t), \\
x_{0}^{\prime}(t) & =\lim _{\Delta \longrightarrow 0} \frac{x_{0}(t+\Delta)-x_{0}(t)}{\Delta} \\
& =\lim _{\Delta \longrightarrow 0} \lim _{m \longrightarrow \infty} \frac{x(t+\Delta+m T, \phi)-x(t+m T, \phi)}{\Delta} \\
& =\lim _{m \longrightarrow \infty} x_{0}^{\prime}(t+m T, \phi) .
\end{aligned}
$$

Hence, we can deduce that $x_{0}(t)$ is a $T$-periodic solution of system (1) since $x(t+m T, \phi)$ is a solution of system (1). 
Now, we prove that $x_{0}(t)$ is a unique $T$-periodic solution of system (1). Let $x_{1}(t)=x_{1}\left(t, \phi_{1}\right)$ and $x_{2}(t)=x_{2}\left(t, \phi_{2}\right)$ be different $T$-periodic solutions of system (1), where $\phi_{1}, \phi_{2} \in C\left([-\rho, 0], \mathbb{R}^{n}\right)$. It follows by (35) that

$$
\begin{aligned}
& \left|x_{1}\left(t, \phi_{1}\right)-x_{2}\left(t, \phi_{2}\right)\right|=\left|x_{1}\left(t+k T, \phi_{1}\right)-x_{2}\left(t+k T, \phi_{2}\right)\right| \\
& \leq \sqrt{\sum_{i=1}^{n} \frac{\lambda_{\max }(P)}{|1-| c_{i}||^{2} \lambda_{\min }(P)} \max _{-\rho \leq s \leq 0}\left\{\left|A\left(\phi_{1}(s)-\phi_{2}(s)\right)\right|\right\} e^{-0.5 k t} \longrightarrow 0 \text { as } k \longrightarrow \infty} .
\end{aligned}
$$

Thus, $x_{1}\left(t, \phi_{1}\right)=x_{2}\left(t, \phi_{2}\right)$ for $t \geq 0$.

Remark 5. In the proof of Theorem 1 , since $z(t)$ is not a continuous $T$-periodic solution, Lemma 1 cannot be used for the proof of Theorem 1. We generalize the results of Lemma 1 to the continuous function space which can be used for the proof of Theorem 1. However, when the parameter $|c| \geq 1$ in (2), we cannot prove the result of Theorem 1. We hope that subsequent researchers can solve the above problems.

Remark 6. Obviously, the periodic solution of system (1) is global asymptotical stability by Theorem 1 . On the contrary, using Lemma 2, we have

$$
\|\phi-\psi\|_{\rho} \leq \sqrt{\sum_{i=1}^{n} \frac{1}{\left.|1-| c_{i}\right|^{2}} \max _{-\rho \leq s \leq 0}\{|A(\phi(s)-\psi(s))|\} .}
$$

If there exists suitable $\widetilde{c}_{i}, i \in \Lambda$, such that

$$
\|\phi-\psi\|_{\rho}=\sqrt{\sum_{i=1}^{n} \frac{1}{\left.|1-| \widetilde{c}_{i}\right|^{2}} \max _{-\rho \leq s \leq 0}\{|A(\phi(s)-\psi(s))|\} .}
$$

Hence, it follows by (35) and (43) that

$$
|y(t, \psi)-x(t, \phi)| \leq \sqrt{\frac{\lambda_{\text {max }}(P)}{\lambda_{\text {min }}(P)}}\|\phi-\psi\|_{\rho} e^{-0.5 \lambda t}, \quad t \geq 0,
$$

and the periodic solution of system (1) is global exponential stability.

Corollary 1. Assume that $B, U$, and $K$ are some known constant matrices. Assume that the assumptions $\left(H_{1}\right)$ and $\left(\mathrm{H}_{2}\right)$ hold. Then, system (1) exists a global asymptotically stable periodic solution if there exist three constants $\varepsilon_{i}>0, i=1,2,3, \quad n \times n$ diagonal matrices $P>0$, and $Q_{i}>0, i=1,2,3$, such that the following inequalities hold:

$$
\left\{\begin{array}{l}
\Xi \geq 0, \\
\varepsilon_{2} P-\sum_{i=1}^{n} \frac{1}{|1-| c_{i}||^{2}} L^{g} Q_{2} L^{g} \geq 0, \\
\varepsilon_{3} P-\sum_{i=1}^{n} \frac{1}{|1-| c_{i}||^{2}} L^{h} Q_{3} L^{h} \geq 0, \\
\varepsilon_{1}-\varepsilon_{2}-\varepsilon_{3} \mu>0,
\end{array}\right.
$$

where

$$
\begin{aligned}
& \Xi=\left(\begin{array}{cccc}
\Xi_{1} & P K & P U & P W \\
* & Q_{1} & 0 & 0 \\
* & * & Q_{2} & 0 \\
* & * & * & Q_{3}
\end{array}\right), \\
& \Xi_{1}=2 P B-\varepsilon_{1} P-\sum_{i=1}^{n} \frac{1}{\left.|1-| c_{i}\right|^{2}} L^{f} Q_{1} L^{f},
\end{aligned}
$$

$L^{f}=\operatorname{diag}\left(l_{1}, \ldots, l_{n}\right)$,

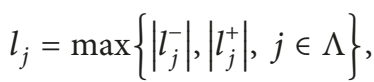

$L^{g}=\operatorname{diag}\left(\sigma_{1}, \ldots, \sigma_{n}\right)$,

$\sigma_{j}=\max \left\{\left|\sigma_{j}^{-}\right|,\left|\sigma_{j}^{+}\right|, j \in \Lambda\right\}$

$L^{h}=\operatorname{diag}\left(v_{1}, \ldots, v_{n}\right)$,

$v_{j}=\max \left\{\left|v_{j}^{-}\right|,\left|v_{j}^{+}\right|, j \in \Lambda\right\}$.

\section{Numerical Example}

In this section, a simulation example is presented for illustrating the usefulness of our main results. Consider a 2neuron neural network (1) with the following parameters: 


$$
\begin{aligned}
& C=\operatorname{diag}\left(\frac{1}{2}, \frac{1}{2}\right) \\
& \sigma=\frac{\pi}{2} \\
& \tau(t)=\mu(t)=0.1, \\
& I(t)=\left(6 \sin \frac{2 \pi}{T} t, 5 \cos \frac{2 \pi}{T} t\right)^{\top}, \\
& B=\left(\begin{array}{ll}
0.9 & 1.2 \\
1.5 & 0.6
\end{array}\right) \text {, } \\
& K \in\left(\begin{array}{cc}
{[0.2,1.4]} & {[-0.8,0.2]} \\
{[-0.2,0.4]} & {[0,1.2]}
\end{array}\right) \\
& U \in\left(\begin{array}{cc}
{[0.3,1.5]} & {[-0.1,0.9]} \\
{[-1.2,-0.6]} & {[0.6,1.8]}
\end{array}\right), \\
& W \in\left(\begin{array}{cc}
{[-1.3,-0.1]} & {[0.1,1.1]} \\
{[-0.9,-0.3]} & {[0.3,1.5]}
\end{array}\right), \\
& f(x)=\tanh (x) \\
& g(x)=0.1 \sin x, \\
& h(x)=3 \cos x .
\end{aligned}
$$

By simple calculation, $\quad L^{f}=I_{2 \times 2}, L^{g}=0.1$ $I_{2 \times 2}, L^{h}=3 I_{2 \times 2}$,

$$
\begin{aligned}
\Gamma_{i} & =\left(\begin{array}{cccc}
\sqrt{0.6} & \sqrt{0.5} & 0 & 0 \\
0 & 0 & \sqrt{0.3} & \sqrt{0.6}
\end{array}\right), \\
\Upsilon_{i} & =\left(\begin{array}{cccc}
\sqrt{0.6} & \sqrt{0.5} & 0 & 0 \\
0 & 0 & \sqrt{0.3} & \sqrt{0.6}
\end{array}\right)^{\top}, \quad i=1,2,3 .
\end{aligned}
$$

Let $\varepsilon_{1}=6, \varepsilon_{2}=2$, and $\varepsilon_{3}=4.2$. We can obtain the following feasible solutions to LMIs in Theorem 1 :

$$
\begin{aligned}
P & =2.8042 I_{2 \times 2}, \\
Q_{1} & =2.7821 I_{2 \times 2}, \\
Q_{2} & =4.4652 I_{2 \times 2}, \\
Q_{3} & =1.6052 I_{2 \times 2}, \\
Q_{4} & =2.9372 I_{4 \times 4}, \\
Q_{5} & =12.1125 I_{4 \times 4}, \\
Q_{6} & =1.5632 I_{4 \times 4} .
\end{aligned}
$$

Hence, all the conditions in Theorem 1 hold, and the 2neuron neural networks (47) have a stationary oscillation with $T$ period. (47) be

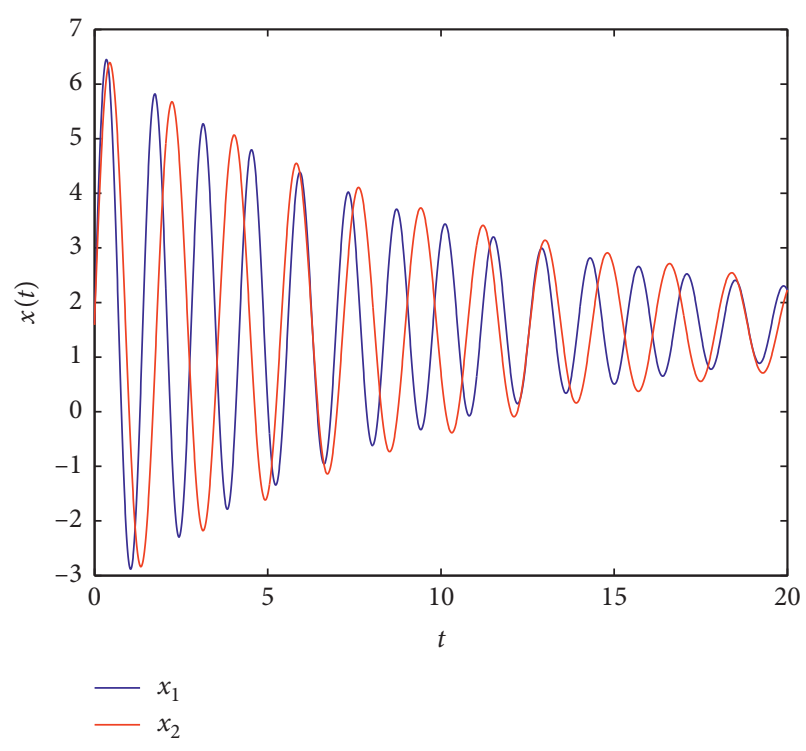

FIGURE 1: State trajectories of system (47) with $T=10$.

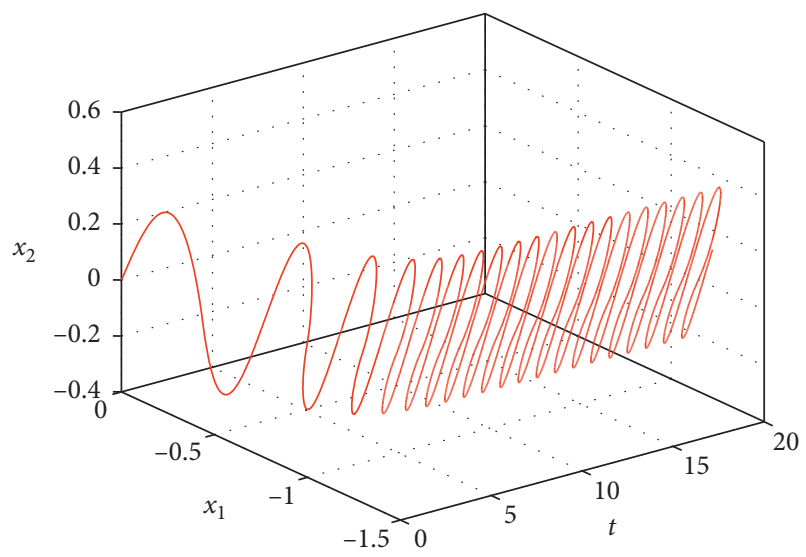

Figure 2: Phase plots of system (47) with $T=10$.

$$
\begin{aligned}
& K_{0}=\left(\begin{array}{cc}
0.2 & -0.6 \\
-0.2,1 & 0.8
\end{array}\right), \\
& U_{0}=\left(\begin{array}{cc}
0.5 & -0.1 \\
-0.5 & 1.7
\end{array}\right), \\
& W_{0}=\left(\begin{array}{cc}
-0.2 & 0.7 \\
-0.3 & 1.2
\end{array}\right) .
\end{aligned}
$$

The corresponding numerical simulations for different $T$ are presented in Figures 1-4. Figure 1 shows the state trajectories of system (47) with the period $T=10$. Figure 2 shows the phase plots of system (47) with the period $T=10$. Figure 3 shows the state trajectories of system (47) with the period $T=0.2$. Figure 4 shows the phase plots of system (47) with the period $T=0.2$. 


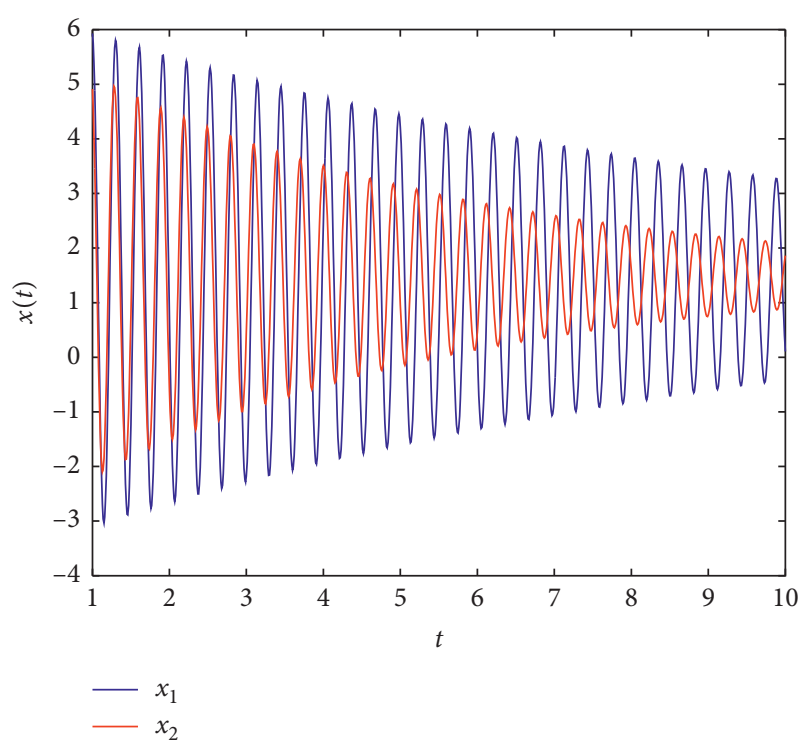

FIgURE 3: State trajectories of system (47) with $T=0.2$.

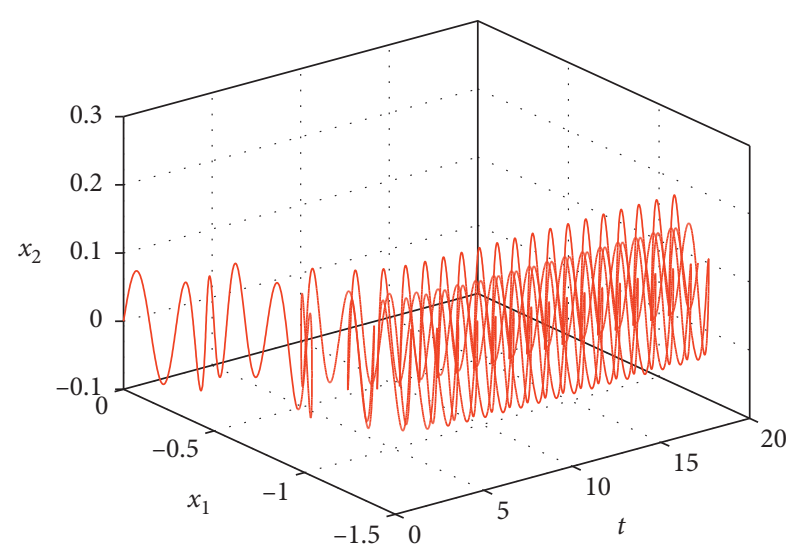

Figure 4: Phase plots of system (47) with $T=0.2$.

Remark 7 . In $[4,5,8,9,11]$, the authors studied the stability problems of some nonneutral interval neural networks with delays. However, there are few results for neutral-type interval neural networks with impulsive delays which is the problem we will solve in the future.

\section{Conclusions}

In this paper, we had investigated the stability problems of neutral-type neural networks with $D$-operator and mixed delays. It is interesting and challenging to extend our results to the stochastic delay system [18], impulsive stochastic delay differential systems [19], semi-Markov switched stochastic systems [20], and stochastic systems with Lévy noise [21].

\section{Data Availability}

The data used to support the findings of this study are included in this paper.

\section{Conflicts of Interest}

The authors declare that they have no conflicts of interests.

\section{Authors' Contributions}

All authors contributed equally to the writing of this paper. All authors read and approved the final manuscript.

\section{Acknowledgments}

This work was supported by the Natural Science Foundation of Jiangsu High Education Institutions of China (Grant no. 17KJB110001).

\section{References}

[1] J. Hale, Theory of Functional Differential Equations, Springer, New York, NY, USA, 1977.

[2] M. Zhang, "Periodic solutions of linear and quasilinear neutral functional differential equations," Journal of Mathematical Analysis and Applications, vol. 189, no. 2, pp. 378-392, 1995.

[3] S. Lu, W. Ge, and Z. Zheng, "Periodic solutions to neutral differential equation with deviating arguments," Applied Mathematics and Computation, vol. 152, no. 1, pp. 17-27, 2004.

[4] M. Syed Ali, N. Gunasekaran, and Q. Zhu, "State estimation of T-S fuzzy delayed neural networks with Markovian jumping parameters using sampled-data control," Fuzzy Sets and Systems, vol. 306, pp. 87-104, 2017.

[5] G. Zong and J. Liu, "New delay-dependent global robust stability conditions for interval neural networks with timevarying delays," Chaos, Solitons \& Fractals, vol. 42, no. 5, pp. 2954-2964, 2009.

[6] Y. Zhang, "Stationary oscillation for cellular neural networks with time delays and impulses," Mathematics and Computers in Simulation, vol. 79, no. 10, pp. 3174-3178, 2009.

[7] Y. Zhang and Q.-G. Wang, "Stationary oscillation for highorder Hopfield neural networks with time delays and impulses," Journal of Computational and Applied Mathematics, vol. 231, no. 1, pp. 473-477, 2009.

[8] H. Zhao, L. Sun, and G. Wang, "Periodic oscillation of discrete-time bidirectional associative memory neural networks," Neurocomputing, vol. 70, no. 16-18, pp. 2924-2930, 2007.

[9] Q. Zhu and J. Cao, "Stability analysis of Markovian jump stochastic BAM neural networks with impulse control and mixed time delays," IEEE Transactions on Neural Networks and Learning Systems, vol. 23, no. 3, pp. 467-479, 2012.

[10] Q. Zhu, J. Cao, and R. Rakkiyappan, "Exponential input-tostate stability of stochastic Cohen-Grossberg neural networks with mixed delays," Nonlinear Dynamics, vol. 79, no. 2, pp. 1085-1098, 2015.

[11] Z. Gui, W. Ge, and X.-S. Yang, "Periodic oscillation for a Hopfield neural networks with neutral delays," Physics Letters A, vol. 364, no. 3-4, pp. 267-273, 2007.

[12] R. Rakkiyappan, P. Balasubramaniam, and J. Cao, "Global exponential stability results for neutral-type impulsive neural networks," Nonlinear Analysis: Real World Applications, vol. 11, no. 1, pp. 122-130, 2010.

[13] Y. Liu, Z. Wang, and X. Liu, "Stability analysis for a class of neutral-type neural networks with Markovian jumping 
parameters and mode-dependent mixed delays," Neurocomputing, vol. 94, pp. 46-53, 2012.

[14] S. Senthilraj, R. Raja, F. Jiang, Q. Zhu, and R. Samidurai, "New delay-interval-dependent stability analysis of neutral type BAM neural networks with successive time delay components," Neurocomputing, vol. 171, pp. 1265-1280, 2016.

[15] R. Raja, Q. Zhu, S. Senthilraj, and R. Samidurai, "Improved stability analysis of uncertain neutral type neural networks with leakage delays and impulsive effects," Applied Mathematics and Computation, vol. 266, pp. 1050-1069, 2015.

[16] S. Cui, T. Zhao, and J. Guo, "Global robust exponential stability for interval neural networks with delay," Chaos, Solitons \& Fractals, vol. 42, no. 3, pp. 1567-1576, 2009.

[17] Z. Wang, H. Shu, J. A. Fang, and X. Liu, "Robust stability for stochastic Hopfield neural networks with time delays," Nonlinear Analysis: Real World Applications, vol. 7, no. 5, pp. 1119-1128, 2006.

[18] Q. Zhu, "Stabilization of stochastic nonlinear delay systems with exogenous disturbances and the event-triggered feedback control," IEEE Transactions on Automatic Control, vol. 64, no. 9, pp. 3764-3771, 2019.

[19] W. Hu, Q. Zhu, and H. R. Karimi, "Some improved Razumikhin stability criteria for impulsive stochastic delay differential systems," IEEE Transactions on Automatic Control, vol. 64, no. 12, pp. 5207-5213, 2019.

[20] B. Wang and Q. Zhu, "Stability analysis of semi-Markov switched stochastic systems," Automatica, vol. 94, pp. 72-80, 2018.

[21] Q. Zhu, "Stability analysis of stochastic delay differential equations with Lévy noise," Systems \& Control Letters, vol. 118, pp. 62-68, 2018. 


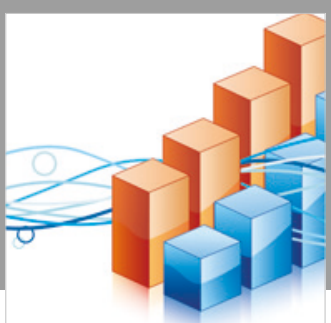

Advances in

Operations Research

\section{-n-m}
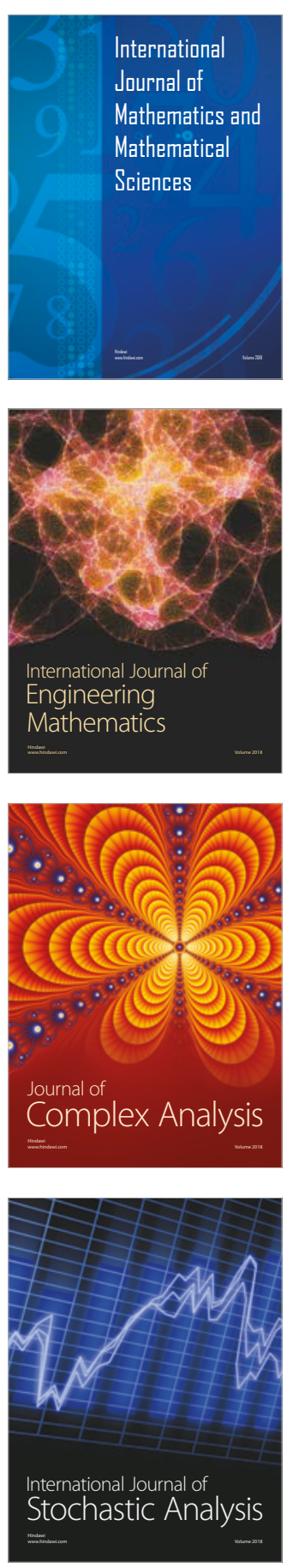
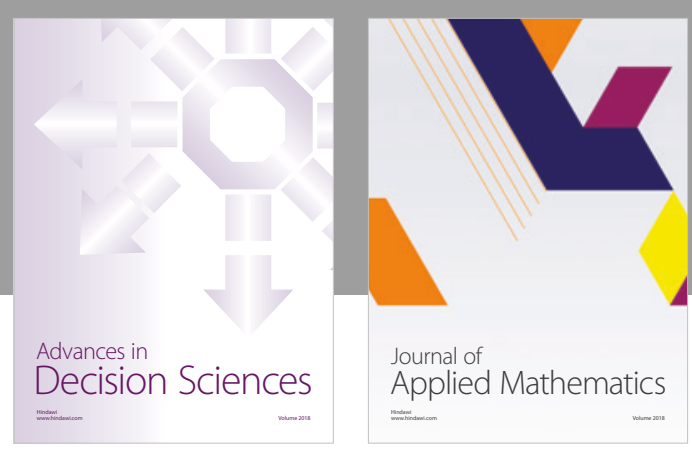

Journal of

Applied Mathematics
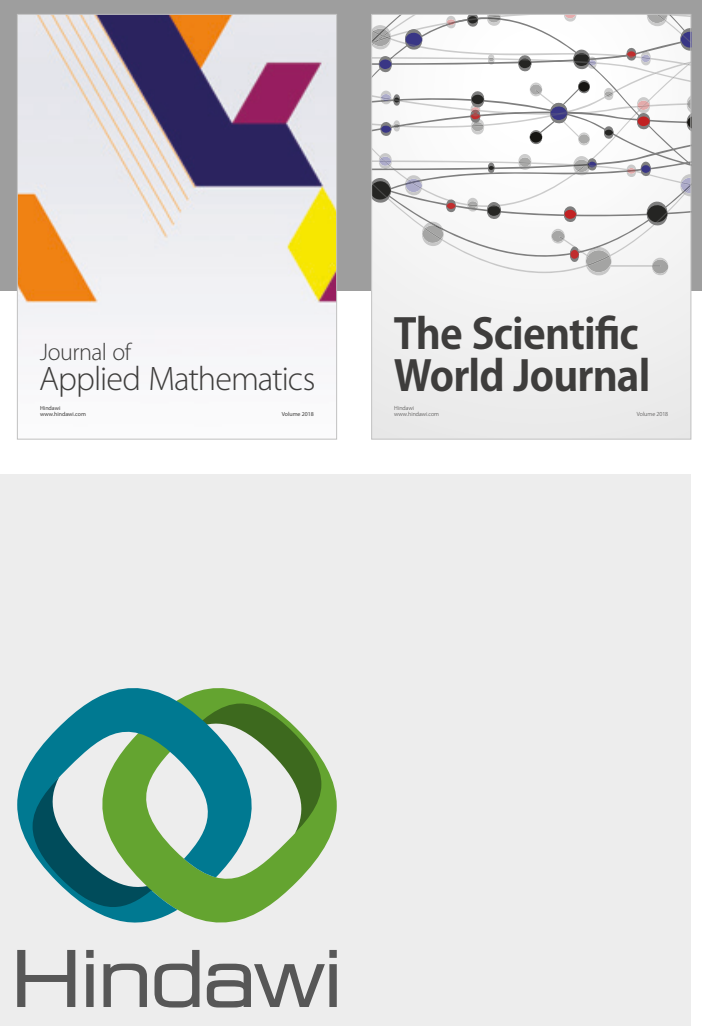

Submit your manuscripts at

www.hindawi.com

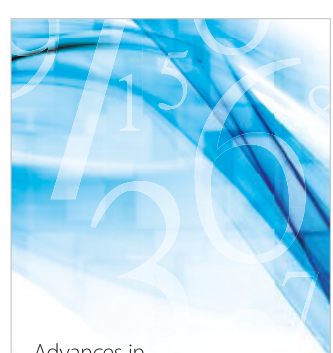

Advances in
Numerical Analysis
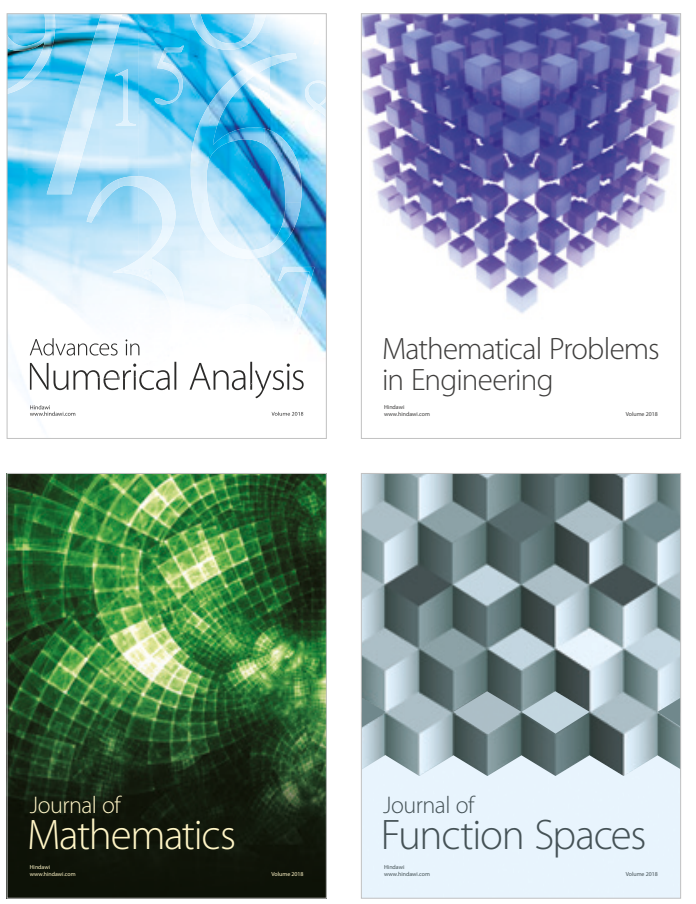

Mathematical Problems in Engineering

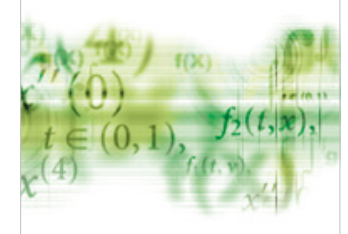

International Journal of

Differential Equations

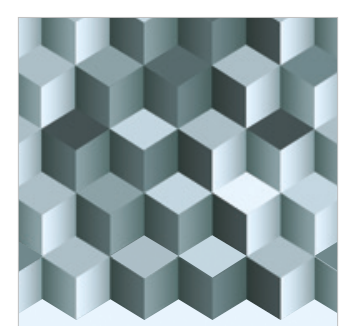

Journal of

Function Spaces

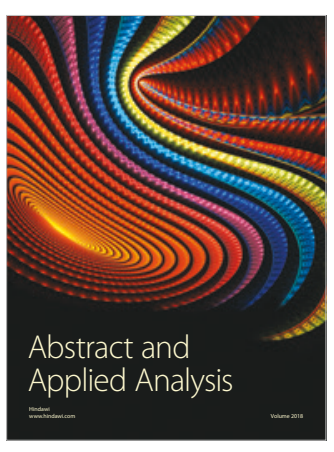

The Scientific

World Journal

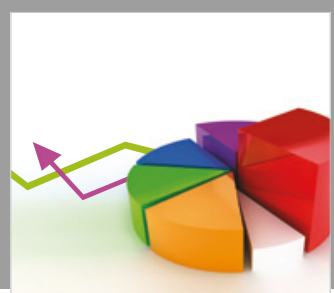

Journal of

Probability and Statistics
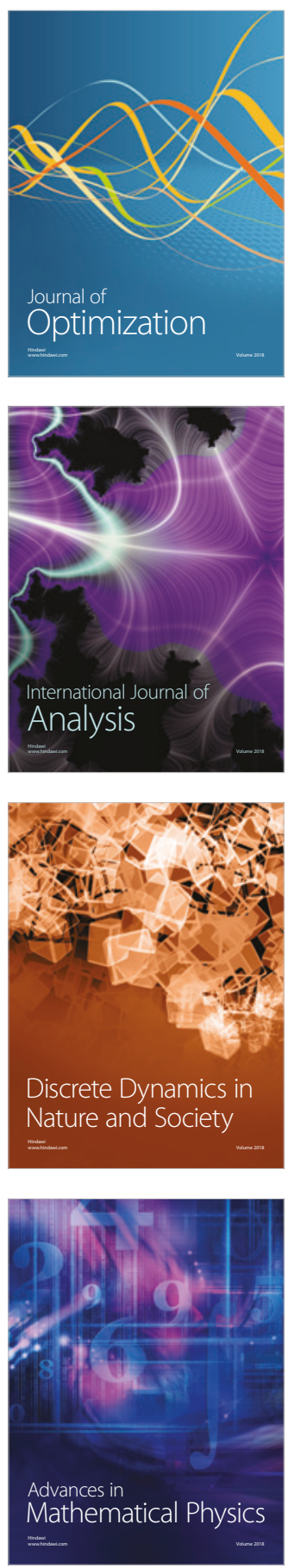